\title{
Tank-Mixing Pesticides without Disasters ${ }^{1}$
}

\author{
Frederick M. Fishel ${ }^{2}$
}

\section{Introduction}

Tank-mixing several pesticides is a common practice for pest control. Tank mixes can consist of much more than just pesticides, such as fertilizers, micronutrients, and adjuvants. The practice offers many advantages, including:

- in some situations, improving the efficacy of products compared to applying products alone;

- increasing the spectrum of pest control by controlling more than one class of pest;

- reducing pesticide resistance cases by combining products with different modes of action;

- reducing soil compaction by reducing the number of trips with heavy equipment across a site; and

- saving time and cost incurred for pesticide application.

\section{Types of Incompatibility}

However, the practice does come with potential disadvantages, including incompatibility. Incompatibility comes in two forms, chemical and physical. Both forms negatively affect mixtures, but in different respects.

Have you ever wondered why a mixture may not have worked as well as anticipated? Chemical incompatibility has its own challenges in that the mixture may appear to be in top quality physically, but its biological activity may be reduced, and it may cause phytotoxicity to desirable plants. A cause of phytotoxicity may be the addition of a crop oil concentrate adjuvant to the mixture. The adjuvant can increase plant cuticle penetration of the pesticide, causing burning to the foliage. Reduced biological activity can be due to mixing certain pesticides with hard water (water with a high concentration of calcium and magnesium ions). Florida is known to have hard water sources. When weakacid herbicides, such as 2,4-D, dicamba, and glyphosate, are mixed with hard water, the calcium and magnesium can bind to the herbicide molecules and render them unable to be taken through the leaf cuticle into the weed's system.

Physical incompatibility occurs when the components in the mixture do not mix well. Separation of components into layers (layering), clumping, excessive foaming, and settling out are all types of incompatibility. This type of incompatibility is particularly frustrating because it causes disruptions in the application. The operator must stop the job and clean out the sprayer system frequently.

1. This document is PI285, one of a series of the Pesticide Information Office, UF/IFAS Extension. Original publication date March 2020. Visit the EDIS website at https://edis.ifas.ufl.edu for the currently supported version of this publication.

2. Frederick M. Fishel, professor, Agronomy Department, and director, Pesticide Information Office; UF/IFAS Extension, Gainesville, FL 32611.

The use of trade names in this publication is solely for the purpose of providing specific information. UF/IFAS does not guarantee or warranty the products named, and references to them in this publication do not signify our approval to the exclusion of other products of suitable composition. Use pesticides safely. Read and follow directions on the manufacturer's label.

The Institute of Food and Agricultural Sciences (IFAS) is an Equal Opportunity Institution authorized to provide research, educational information and other services

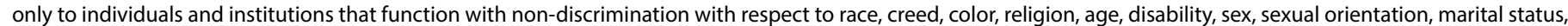

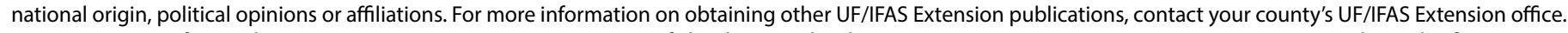
U.S. Department of Agriculture, UF/IFAS Extension Service, University of Florida, IFAS, Florida A \& M University Cooperative Extension Program, and Boards of County Commissioners Cooperating. Nick T. Place, dean for UF/IFAS Extension. 
Knowing When to Mix and When Not to Mix: The Physical Compatibility Jar Test

Rather than make a complete mess in the spray tank, conduct a relatively quick, small-scale jar test, using jars with a capacity of one quart. Many product labels in their mixing directions will instruct the user to perform a physical compatibility "jar test" and provide directions on the proper mixing sequence of various formulations, adjuvants, and fertilizers (Figure 1). Reading the label(s) for this information should always take place before haphazardly pouring product(s) into jars. The amount to add into the jar should be proportionate to the amount that will be used in the actual mix tank. It will require conversion to the correct measurement units, such as milliliters, ounces, and teaspoons. Some product labels will state how much of each product to use in conducting a jar test. An important consideration is to establish the carrier used in the mixwater or liquid fertilizer. It may be most desirable to use two jars, one with and one without a compatibility agent. This is especially important if a liquid fertilizer will serve as the carrier. Fill each jar to one-half capacity with carrier. Add the mix components in order as recommended by the label(s) into the jars. Cap the jars, gently shake or invert, then let stand for 15 to 30 minutes. If physical incompatibility signs are noticed, reshake and let stand again. Check again for physical incompatibility to determine if the mixture is suitable for large-scale use. However, be aware that the jar test cannot detect chemical incompatibility.

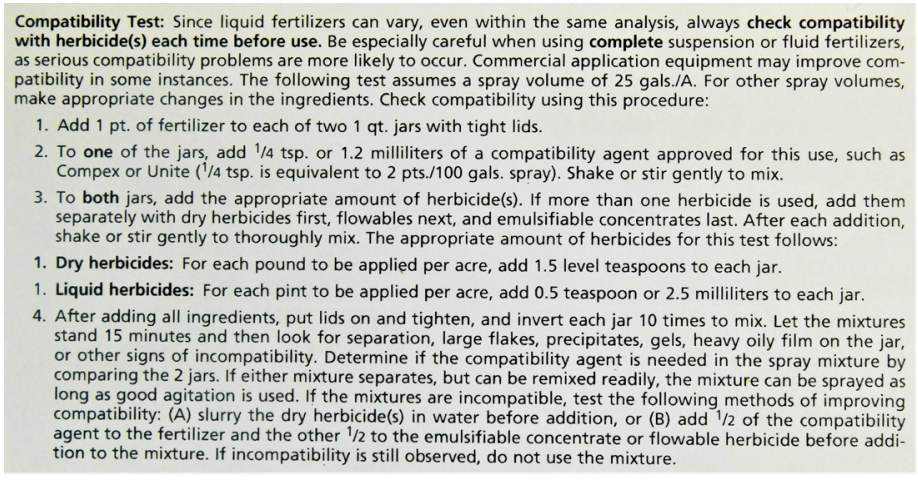

Figure 1. Mixing instructions to perform a compatibility jar test.

Credits: UF/IFAS Pesticide Information Office

\section{Making the Tank Mix}

If water is the carrier, these are the general steps:

1. Read all product labels carefully.

2. Shake all products thoroughly as some product ingredients will layer while held in storage.
3. Fill tank with $50 \%$ of the total volume of water to be used.

4. Start agitation before adding the first product, and continue agitation throughout the mixing process.

5. Add products in order of their formulations (see following section). If you have questions with specifics, such as the product's formulation, the manufacturer can provide insight; many labels don't clearly state the formulation.

6. Add remaining water.

If water is the carrier, add products in this order:

1. Ammonium sulfate (AMS).

2. Water-soluble packets.

3. Dry formulations, such as wettable powders and waterdispersible granules. Always wait 3 to 5 minutes following each dry product that is added. This allows them adequate time to dissolve.

4. Dry antidrift agents (drift retardants).

5. Compatibility agents and antifoamers. Before continuing, wait a couple of minutes to allow them time to become active.

6. Dispersed liquid formulations. These include flowables and suspension concentrates.

7. Liquid antidrift agents.

8. Remaining liquid formulations, including emulsifiable concentrates and solutions.

9. Adjuvants, including crop oil concentrates, non-ionic surfactants, and methylated seed oils.

10. Micronutrients and liquid fertilizers.

If liquid fertilizer is the carrier, these are the general steps:

1. Read all labels.

2. Shake all products thoroughly; some product ingredients will layer while held in storage.

3. Fill tank with $50 \%$ of the total volume of liquid fertilizer to be used. 
4. Start agitation before adding the first product and continue agitation throughout the mixing process.

5. Add products in order of their formulations (see following section). For best results with dry products, form a preslurry with a small volume of water before adding into the tank. If you have questions with specifics, such as the product's formulation, the manufacturer can provide insight; many labels don't clearly state the formulation.

\section{Add remaining fertilizer.}

If liquid fertilizer is the carrier, add products in this order:

1. Make a preslurry of water with water-soluble packages and other dry formulations.

2. Make a preslurry of water with dry antidrift agents (drift retardants).

3. Compatibility agents and antifoamers. Before continuing, wait a couple of minutes to allow them time to become active.

4. Dispersed liquid formulations. These include flowables and suspension concentrates.

5. Liquid drift retardants, such as polymer-based products.

6. Remaining liquid formulations, including oil-based emulsifiable concentrates and solutions.

7. Adjuvants, including crop oil concentrates, non-ionic surfactants, and methylated seed oils.

\section{Micronutrients.}

These are general tank-mixing procedures and don't pertain to every specific product or situation. Always defer to the label for the specifics.

\section{Commercial Tank-Mixing Tools}

There are commercial tank-mixing tools available to make mixing and jar-testing easier.

Several apps are readily available to download for iPhone or Android, such as Precision Laboratories (http://www. mixtankapp.com/onlineapp/index.php). They are designed to assist applicators with the proper tank-mixing sequence of crop protection products. The database on these apps contain multiple products that the applicator can select, including adjuvants, fertilizers, and all major classes of pesticides. The user simply selects the various products that will be in the tank mix, and the app provides an output of the correct mixing order without incompatibility problems.

There are also commercially available compatibility test kits (Figure 2). These can replace the need to keep on hand an elaborate series of measuring cups, cylinders, tablespoons, and teaspoons. One of the most useful is based on a collection of 1 milliliter pipettes. Each pipette represents 1 gallon of liquid and is marked in $1 / 4$-milliliter increments, each mark representing 1 quart. One pipette that comes with the kit contains a scoop with the end of the bulb cut open to measure dry materials. A single scoop represents 8.5 pounds, which is a standard rate to add ammonium sulfate, a common water conditioner. The clear plastic polyvinyl chloride containers that come with the kit hold 120 milliliters but are marked at the 100-milliliter level. One hundred milliliters corresponds to 100 gallons of water. The mixing container is calibrated based on the number of acres a 100-gallon tank will cover. After each product is added, the user inverts the test container to ensure proper mixing. After approximately 30 minutes of waiting, the container can be checked for possible physical incompatibility. The kit recommends letting mixtures sit overnight.

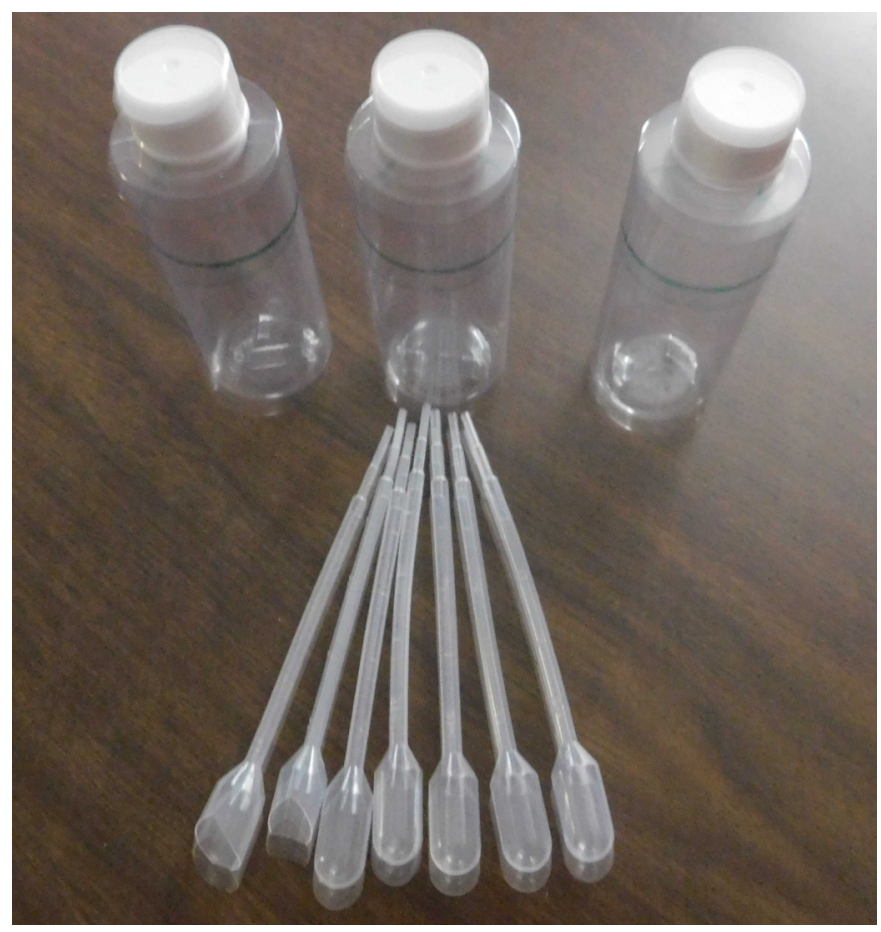

Figure 2. Commercially available compatibility test kit. Credits: UF/IFAS Pesticide Information Office

Table 1 provides an example to demonstrate how this kit works. 
Table 1. Example product calculation for commercial compatibility test kit.

\begin{tabular}{|l|c|c|c|c|c|}
\hline \multicolumn{1}{|c|}{ Product } & Use rate & Conversion factor & Total/100 gal & $\begin{array}{c}\text { For 100 gal } \\
\text { converted rates }\end{array}$ & Total/100 ml \\
\hline RoundupPowerMAX & $40 \mathrm{oz} / \mathrm{acre}$ & $\times 6.67$ & $=266.8 \mathrm{oz}$ & $/ 32=8.3 \mathrm{qt}$ & $/ 4=2.1 \mathrm{ml}$ \\
\hline 2,4-D LV4 & $1 \mathrm{pt} / \mathrm{acre}$ & $\times 6.67$ & $=6.67 \mathrm{pt}$ & $/ 2=3.3 \mathrm{qt}$ & $/ 4=0.8 \mathrm{ml}$ \\
\hline Atrazine 4L & $1.5 \mathrm{qt} / \mathrm{acre}$ & $\times 6.67$ & $=10.01 \mathrm{qt}$ & $10.0 \mathrm{qt}$ & $/ 4=2.5 \mathrm{ml}$ \\
\hline Crop Oil & $1 \% \mathrm{v} / \mathrm{v}$ & $\mathrm{NA}$ & $4 \mathrm{qt}$ & $4 \mathrm{qt}$ & $/ 4=1.0 \mathrm{ml}$ \\
\hline Ammonium Sulfate & $8.5 \mathrm{lb} / 100 \mathrm{gal}$ & $\mathrm{NA}$ & $8.5 \mathrm{lb}$ & $8.5 \mathrm{lb}$ & $1 \mathrm{scoop}$ \\
\hline
\end{tabular}

Products and rates to be included in the mix are shown in columns 1 and 2, respectively. The products are not necessarily shown in the correct mixing sequence.

In this example, a total of 100 gallons of spray mix will be made up using water as the carrier, and the sprayer is calibrated to make the application delivered at 15 gallons per acre (gpa). Therefore:

100 gallons $\div 15$ gpa $=6.67$ acres treated per load of mix (conversion factor used in column 3 ) for determining rates of product per acre.

The total amount of each product is multiplied by the conversion factor to determine the total for each product needed to mix into 100 gallons of water (column 4).

Because these rates are not in the same units and because each measuring pipette is divided into quart increments, the rates are converted to use quart units (column 5). Then the quart rate is divided by 4 (number of quarts in one gallon). This will determine the number of $\mathrm{ml}$ to add to each 100 -ml container.

Because there is a total volume of $6.4 \mathrm{ml}$ of product, after filling half of the $100-\mathrm{ml}$ container with water, add the product and then fill to the $100-\mathrm{ml}$ level (green marking) with the remaining volume of water.

Before continuing, ask yourself: what is the correct mixing sequence for the products shown in Table 1? The answer is below.

\section{Ammonium sulfate}

\section{Atrazine $4 \mathrm{~L}$}

\section{3.,4-D LV 4}

4. Roundup

5. Crop oil concentrate

\section{Conclusions}

Pest managers are working with many potential tank-mix combinations of products. Formulation chemistry has become more complex, not simpler. Chemical and physical incompatibility are always pitfalls faced by applicators, who must use caution when combining products. Mixing various products in the proper order is paramount to achieving a successful application and meeting the goal of maximizing pest control efficacy. Undertaking precautionary measures, such as jar testing, prior to spraying can assist in minimizing the disaster of pest control failures or interrupting the application by having to stop and clean out the sprayer system. Always read and follow all product labels carefully! 